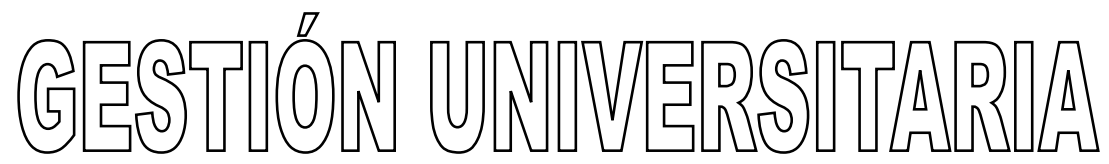

\title{
EL PROGRAMA UNIVERSIDAD EMPRENDEDORA (PUE) EN NICARAGUA: ACTIVIDADES, PRODUCTOS Y RESULTADOS
}

\section{INNOVATIVE UNIVERSITY PROGRAM (IUP) IN NICARAGUA: ACTIVITIES, PRODUCTS AND RESULTS}

\author{
Aleman Freddy ${ }^{1}$, Scheinberg Sari² \\ ${ }^{1} \mathrm{PhD}$, Director de Investigación, Extensión y Posgrado, Universidad Nacional Agraria, freddy@una.edu.ni \\ ${ }^{2} \mathrm{PhD}$. Universidad Tecnológica de Chalmers - Centro de Estudi de Propiedad Intelectual (CIP). \\ sari.scheinberg@chalmers.edu.se
}

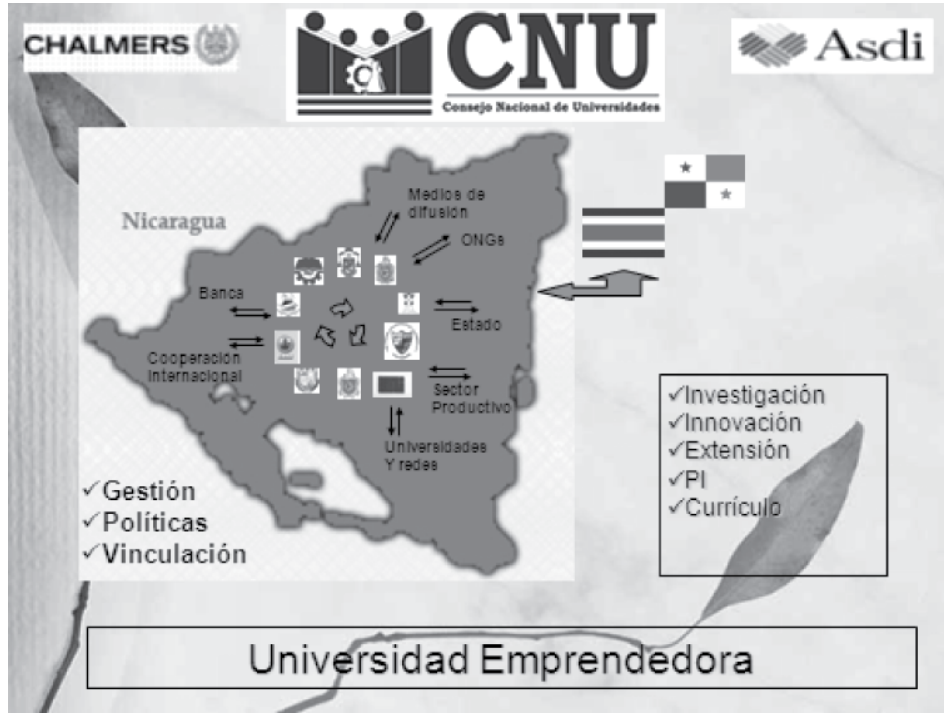

\section{RESUMEN}

Las diez Universidades que conforman el Consejo Nacional de Universidades (CNU) en Nicaragua participaron en un programa (2007 a 2010) orientado a desarrollar el papel y la contribución de las Universidades en los procesos de innovación. El programa fue financiado por la Agencia Sueca para el Desarrollo Internacional (Asdi), y se basó en una metodología de acción-aprendizaje, en el cual las universidades compartían sus experiencias, competencias, estrategias y actividades entre ellas, así como, con otros actores claves en los sistemas de innovación. La Universidad Tecnológica de Chalmers, a través de la fundación Stiftelsen Chalmers Industriteknik (CIP) fue la contraparte sueca, con participación de investigadores de la escuela de tecnologías, gestión y economía, y el centro para estudios de propiedad intelectual (CIP). Los objetivos del programa fueron de extrema importancia para el desarrollo de las Universidades Nicaragüenses, y
The ten universities that comprise the National Council of Universities (CNU) in Nicaragua participated in a program (2007 to 2010) aimed at developing the role and contribution of universities in a process of innovation. The program was funded by the Swedish International Development Agency (Sida), and was based on actionlearning, in which universities shared their experiences, skills, strategies and activities between them and with other key groups in the innovation systems. Chalmers University of Technology, through the foundation Stiftelsen Chalmers Industriteknik (CIP) was the Swedish counterpart, involving researchers from the school of technology, management and economics, and the Center for Intellectual Property studies (CIP). The program's goals were of extreme importance for the development of the Nicaraguan Universities, and are similar to the challenges that universities in the world are facing at present. In search of improvement of innovative process at the universities, all the 
en todo el mundo enfrentan en el momento actual. En busca de la mejora de los procesos innovadores de las Universidades se definieron los siguientes objetivos: Desarrollar los roles y las responsabilidades que el CNU y las Universidades poseen en el sistema actual y futuro de innovación en Nicaragua, en asociación con otros grupos de claves en Nicaragua; generar las políticas que permitan a la Universidad incluir la innovación en su misión y prácticas; evaluar y mejorar el proceso de manejo de la investigación, incluyendo planes, diseños, métodos, construcción de relaciones y prácticas gerenciales que apoyarán la aplicabilidad e integración de las actividades de investigación y los resultados en la sociedad; desarrollar funciones, procesos y estructuras en las Universidades, que permitan que la innovación y el emprendedurismo sean integrados en su visión y prácticas, particularmente en el proceso de "investigación para el mercado". Desarrollar a fondo el rol y la profesión del investigador, que incluyan contratos e incentivos para asegurar el desarrollo continuo de este grupo profesional desde una perspectiva de investigación e innovación; desarrollar una atmósfera y cultura en las Universidades (y el CNU) que estimulen transparencia, cooperación y aprendizaje, por medio del desarrollo de métodos de trabajo sistemáticos y conscientes, reflexionando y compartiendo; desarrollar habilidades y estrategias para proteger y extraer valor de la propiedad intelectual, basada en nuevos conocimientos y tecnologías generadas en las universidades, y por ultimo formar personal especializado a nivel de maestría, con el propósito de desarrollar habilidades locales en el área de propiedad intelectual y gerencia del capital intelectual. Este escrito presenta las acciones realizadas, los resultados y las conclusiones del programa.

Palabras clave: Universidad innovadora, innovación, gestión universitaria, transferencia tecnológica, propiedad intelectual, emprendedurismo. actors involved identified the following objectives: to develop the roles and responsibilities that the CNU and the universities have in the current and future system of innovation in Nicaragua, in partnership with other key groups; generate the policies to enable the University to include innovation in their mission and practices; to evaluate and improve the process of researchmanagement, including plans, designs methods, building relationships and management practices that will support the applicability and integration of research activities and results into society; to develop functions, processes and structures in the universities, that enable innovation and entrepreneurship be integrated into his vision and practices, particularly in the process of " research to market". Moreover, the entrepreneurial university program aims to: develop in depth the role and profession of the researcher, and the contracts and incentives to ensure the continued development of this professional group from a perspective of research and innovation; to develop an atmosphere and culture at the Universities (and the $\mathrm{CNU}$ ) to encourage more transparency, cooperation and learning, by developing a more systematic and conscious approach, reflecting and sharing, to develop skills and strategies to protect and extract value from intellectual property, and train personnel at Masters level to develop local skills in the area of intellectual property and Intellectual Capital Management. This paper presents the actions taken, results and conclusions of the program.
$\mathrm{E}$ 1 pueblo y reinado de Suecia, a través de la Agencia Sueca para el Desarrollo Internacional (Asdi) ha sido un soporte importante para las Universidades públicas nicaragüenses por un periodo aproximado de treinta años. Esta colaboración se enfocó en el fortalecimiento de las capacidades de investigación en las universidades, cooperación técnica, becas para investigadores y estudiantes, programas de transferencia de conocimientos, conferencias y programas de capacitación, entre otros.

A pesar de la evidencia de logros sustanciales producto de la cooperación, la industria, el gobierno y los proveedores de fondos, en su momento, expresaron su preocupación en relación a la invisibilidad de los resultados de la investigación de las Universidades (Johansson, 2009).Nadie parecía saber concretamente que se ha hecho con la enorme cantidad de conocimientos, tecnologías y aprendizajes generados por tantos años de actividades de investigación (Alange, S, \& S Scheinberg, 2005)

Ante la problemática planteada cabían las siguientes preguntas ¿Para qué han servido los resultados de investigación de dos décadas de cooperación SueciaNicaragua? ¿Cuál ha sido el impacto de esta colaboración 
en investigación? Lo anterior obligó a las autoridades universitarias a hacer una mirada retrospectiva $\mathrm{y}$ preguntarse ¿Qué cambios, estrategias, procesos, son necesario para permitir a las Universidades transferir los resultados de las investigaciones a la sociedad y a la industria? ¿Qué nuevas tareas y roles debemos jugar para permitir la transformación de los estudiantes en emprendedores (no solo empleados), y que los resultados de las investigaciones y de los procesos que se desarrollan en las Universidades no se presenten únicamente en artículos científicos y publicaciones, sino también en productos y servicios innovadores que creen cambio en la sociedad.

Con el propósito de contribuir a la solución de los problemas planteados se diseñó el Programa Universidad Emprendedora (2007-2010), bajo una metodología de acción-aprendizaje cuyo propósito fue apoyar al Consejo Nacional de Universidades (CNU) y las Universidades que lo conforman a convertirse en "universidades innovadoras". Además de las Universidades del CNU, en este programa participaron miembros de cámaras empresariales (COSEP), empresarios (Jaleas Callejas, Banca Nacional, Medios de comunicación), organizaciones no gubernamentales (FUNICA, CIAN) y actores del Gobierno (Presidencia de la República, CONICYT, MIFIC-PAIT, MIFIC-RPI, $\mathrm{CPC}$ ), quienes tienen particular interés en la creación y funcionamiento del Sistema Nacional de Innovación.

El programa fue orientado a desarrollar el papel y la contribución de las Universidades en los procesos de innovación, fue financiado por la Agencia Sueca para el Desarrollo Internacional (Asdi), y se basó en acciónaprendizaje, en el cual las universidades compartían sus experiencias, competencias, estrategias y actividades entre ellas, así como, con los otros grupos claves en los sistemas de innovación.

La universidad tecnológica de Chalmers, a través de la fundación Stiftelsen Chalmers Industriteknik (CIP) fue la contraparte sueca, con participación de investigadores de la escuela de tecnologías, gestión y economía, y el centro para estudios de propiedad intelectual (CIP). Los objetivos del programa fueron de extrema importancia para el desarrollo de las Universidades Nicaragüenses, y no estaban distantes de los retos y desafíos que las Universidades en todo el mundo enfrentan en el momento actual.

El proceso de trabajo del programa Universidad Emprendedora (PUE). La metodología de trabajo del PUE se basó en la organización de mecanismos de consulta y difusión, tales como talleres con académicos de las diez universidades y actores claves del sistema de investigación e innovación, sesiones de capacitación con expertos nacionales e internacionales, reuniones con grupos de interés para difundir los resultados de los programas y talleres y la preparación de material impreso. Este conjunto de actividades constituyó el núcleo del programa, y ayudó construir una red de colaboración entre las universidades y diferentes sectores de la sociedad nicaragüense.

Entre las actividades de divulgación se encuentra la organización de siete talleres con participación de académicos de las diez universidades y actores claves del sistema de innovación de Nicaragua, donde se discutieron aspectos como los mecanismos a implementar para que una Universidad sea innovadora, como debería ser organizada y estructurada y cómo deben ser las relaciones de trabajo con sus socios claves, etc. De igual forma se establecieron reuniones de alto nivel con autoridades nacionales para obtener apoyo político, así como con representantes del sector financiero, la industria y los medios de comunicación.

Por otro lado, en el proceso de trabajo se participó en un viaje de intercambio (benchmarking), donde los líderes del programa en cada una de las diez universidades visitaron Costa Rica y Panamá con el objetivo de conocer la experiencia de las universidades públicas de países relativamente más avanzados en la organización de sus sistemas de innovación. De igual forma los avances del programa fueron difundidos en conferencias de prensa y entrevistas a los medios de comunicación locales. La Tabla 1, muestra las actividades de aprendizaje, cuando fue realizada y los temas claves discutidos

Evaluación de medio término. Con un enfoque de evaluación continua, y para profundizar en un proceso de reflexión y retroalimentación, después de trascurridos año medio del programa se realizó en cada una de las Universidades una evaluación de medio término. Líderes del programa en cada una de las universidades respondieron un formulario que contenía los objetivos, acciones y planes. Este mecanismo permitió al equipo líder estar conscientes de los logros del programa en cada una de las universidades, y aplicar los ajustes necesarios con el fin de re-direccionar las metas, si ese fuere el caso. Con este ejercicio, se conoció acerca de lo que había que mejorar, y los pasos a seguir para el futro inmediato. Los resultados de esta evaluación fueron dados a conocer a las universidades como una retroalimentación que permitiera establecer metas y prioridades para los meses restantes en el programa.

Un aspecto positivo de las actividades realizadas fue la realización de informes trimestrales del avance de las actividades implementadas en cada una de las universidades, en correspondencia con los objetivos del programa. 
Tabla 1. Talleres y temas claves desarrollados en los tres años del programa Universidad Emprendedora

\begin{tabular}{lll}
\hline Actividad & Realización & \multicolumn{1}{c}{ Tema clave desarrollados } \\
\hline Taller 1 & Julio 2007 & $\begin{array}{l}\text { Conceptos innovadores en una universidad, tendencias mundiales, la } \\
\text { innovación, el ciclo de la experiencia (forma sistemática de trabajar) }\end{array}$ \\
Taller 2 & febrero 2008 & $\begin{array}{l}\text { Políticas universitarias de investigación, PI, innovación, espíritu } \\
\text { empresarial. Teorías sobre la construcción de relaciones para la creación de } \\
\text { alianzas estratégicas y relaciones. }\end{array}$
\end{tabular}

Taller 3 febrero 2008

Sistemas de innovación, construcción de sistemas de innovación, y la resolución de problemas

Taller $4 \quad$ mayo 2008

Función de transferencia de tecnología, evaluación de relaciones exitosas, alianzas estratégicas, cómo la industria percibe y utiliza las alianzas estratégicas con la universidad

Benchmarking julio de 2008 Costa Rica y Panamá. Conocer la experiencia del sistema de innovación

Taller in situ noviembre 2008 Actividad en cada una de las Universidades, para evaluar el avance del programa y definir prioridades inmediatas

Taller 5 feb 2009

IP y la política de innovación, alianzas estratégicas, las presentaciones de los líderes de la industria nicaragüense y la presentación de casos

Taller $6 \quad$ julio de 2009

Integración y sostenibilidad, revisar el estado del programa y los objetivos y planificación para el año de salida del, programa

Taller 7 Junio $2010 \quad$ Revisión de los resultados obtenidos y cierre, conferencia nacional "Nicaragua innova".

\section{Los productos del programa Universidad} Emprendedora. El PUE se orientó a fortalecer el papel de la Universidad, sus competencias, estructuras, las prácticas de gestión y las relaciones con los actores claves de los sistemas de innovación, con el fin de generar conocimiento y estrechar la cooperación en pro de la innovación, de tal forma que ésta beneficie a la sociedad nicaragüense.

En el marco del programa, en las Universidades se desarrollaron acciones orientadas a la introducción de conceptos y un marco conceptual para entender y promover la innovación, que permitieran la introducción de reformas universitarias, y el desarrollo de funciones, procesos y estructuras para la innovación.

También, se trabajó en el impulso de acciones orientadas a transformar los roles y las responsabilidades del CNU y las Universidades que lo conforman en el sistema actual y futuro de innovación en Nicaragua, de igual forma se desarrolló un proceso participativo para al análisis del papel de los restantes actores claves en el sistema. Es por ello que las Universidades que conforman el CNU y el CNU como tal inicio un acercamiento con instituciones de gobierno, la iniciativa privada, el sector financiero, los medios de comunicación, los gremios (UNAG, UPANIC), etc., enfatizando sobre la necesidad de desarrollar sinergias para un mejor impacto de las acciones de ciencia y tecnología desarrolladas en las instituciones académicas.

Además, el PUE enfatizó la creación de políticas y estrategias para que la innovación fuera una realidad en las Universidades Nicaragüenses, así como la inclusión de la transferencia de tecnología y la propiedad intelectual en la misión y práctica universitaria. Uno de los aspectos más influenciados por el PUE en las Universidades Nicaragüenses fue el proceso de gestión de la cadena de generación de conocimiento. Se trabajó decisivamente 
en influenciar planes, diseños, métodos y prácticas de gestión que permitieran la aplicación e integración de las actividades de investigación y los resultados de las mismas en la creación de bienestar para la sociedad. De igual forma se trabajó en desarrollar funciones, procesos y estructuras en las universidades con el fin de crear una orientación innovadora y emprendedora en la visión y prácticas universitarias, sobre todo en la gestión del conocimiento y en la creación de valor a partir de los productos de las investigaciones que desarrollan las Universidades.

Todos los aspectos referidos no pueden ser logrados si no se revisan aspectos claves en el Sistema Universitario de generación de conocimientos, siendo uno de ellos la carrera del investigador. El PUE enfatizó sobre el papel y la carrera del investigador, sobre la necesidad de espacios de tiempo e incentivos para asegurar el desarrollo continuo de los académicos desde una perspectiva de investigación e innovación. Pero también, a través de este programa, en el CNU y en las Universidades, se propició un ambiente y una cultura que promoviera mayor transparencia, intercambio y aprendizaje mediante mejores métodos de trabajo a través de reflexión y cooperación entre los actores claves de los sistemas de investigación e innovación.

Las Universidades organizaron cursos y entrenamientos cortos sobre la gestión de la propiedad intelectual, en ellas participaron autoridades y académicos. En estas actividades se trabajó en estrecha colaboración con el Ministerio de Economía (MIFIC), específicamente con la oficina de Propiedad Intelectual.

Hasta hace unos cinco años en las Universidades Nicaragüenses no existía cultura de protección de los productos generados de los procesos de investigación. A través del PUE se trabajó decididamente en sensibilizar a la comunidad Universitaria sobre la importancia de proteger los activos intangibles de propiedad intelectual. Estos procesos llevaron a la definición de acciones institucionales y al desarrollo de estrategias y prácticas de gestión para evaluar, proteger y crear valor de la propiedad intelectual sobre la base de nuevos conocimientos, servicios, productos y tecnologías generados en la universidad.

Los productos se resumen de la siguiente forma:

- Definición de políticas y estrategias para incluir la innovación en la misión y prácticas de las universidades

- Mejora en los procesos de gestión de la investigación y la innovación

- Desarrollo de funciones de transferencia tecnológica en las universidades
- Promoción de la carrera del investigador en las Universidades.

- Integración de actividades fragmentadas en un solo objetivo de fortalecer la función de extensión de la universidad y la colaboración entre universidades.

- Promoción de una plataforma de diálogo entre el sector privado, las universidades y el sector público

- Promoción de un ambiente y cultura que promueve el intercambio y el aprendizaje con otros actores claves del sistema

- Desarrollo de estrategias y mecanismos de interacción entre universidades y actores claves de la sociedad.

- Mejora en la gestión de la protección a través de la propiedad intelectual de conocimientos servicios, productos y tecnologías generadas.

- Soporte para la formación, a nivel de maestría, de estudiantes nicaragüenses en gestión del capital intelectual.

- Fortalecemiento de los currículos de grado y posgrado en investigación y emprendimiento.

- Aprendizaje a partir de experiencias de las Universidades del CNU, acerca de buenas prácticas de gestión de investigación e innovación, y retomar la experiencia para el trabajo futuro.

- Hacer visible lo que producen las Universidades, mostrar nuestras potencialidades en materia de servicios para la sociedad.

- Lograr un lenguaje común y apropiación de los conceptos de innovación, espíritu empresarial, y mejora de los servicios para atender las necesidades del sector privado.

- Promocion de un marco común para alinear los intereses de investigación entre universidades y empresas.

- Contribuir, junto a un grupo de expertos, y junto al Consejo Nacional de Ciencia y Tecnología (CONICYT), a plasmar una propuesta de proyecto de ley de ciencia y tecnología e innovación.

- Proporcionar entrenamiento para el desarrollo de políticas de propiedad intelectual, innovación e investigación.

- Desarrollo de procesos orientados al establecimiento de políticas y normas de propiedad intelectual.

- Capacidades humanas desarrolladas (autoridades y académicos) mediante entrenamientos (cursos) sobre gestión de propiedad intelectual.

- Desarrollo de procesos orientados a identificar productos y servicios sujetos de protección a través de la propiedad intelectual. 
Principales resultados del Programa Universidad emprendedora. Los resultados generados por las actividades y productos descritos fueron evidenciados a través de evaluaciones trimestrales del desempeño de programa, una evaluación de medio término y una evaluación final del mismo. En lo que sigue, se presentan los resultados obtenidos sin importar a que objetivo se circunscribe. Se enfatiza sobre las lecciones aprendidas y las líneas de acción que deberían ser llenadas para continuar con el proceso demarcado por el programa.

Un resultado a resaltar es creación de capacidades en los individuos. Por ello, el PUE permitió la capacitación de gran cantidad de académicos de las Universidades nicaragüenses pertenecientes al CNU en los diferentes eslabones de la cadena de generación de conocimientos, se apoyó la formación a nivel de maestría de cinco nicaragüenses en Suecia, y la capacitación de académicos nicaragüenses en evaluación de ideas de negocios y estudios de factibilidad en Nicaragua, con el fin de desarrollar competencias locales en gestión del capital intelectual y propiedad intelectual. Los académicos que recibieron formación a nivel de maestría provinieron de Universidades nicaragüenses, y están trabajando en Nicaragua.

La lógica detrás de PUE fue incrementar la capacidad y el liderazgo de las universidades Nicaragüenses para colaborar con otros actores claves del sistema, con el fin de promover la innovación, generar sinergias y mejorar el sistema productivo, con la participación de todos. El impacto PUE en las Universidades Nicaragüenses es perceptible a través de la colaboración en investigación y a través de como las ideas difundidas por el programa están impregnando y contribuyendo a transformar las estructuras institucionales, mediante formas de interactuar con otras actores claves, y el cambio de las aptitudes de los investigadores universitarios, autoridades, instituciones públicas y el sector privado, acerca de conceptos como la innovación, el espíritu empresarial, la propiedad intelectual y la colaboración en la investigación, entre otros.

El PUE contribuyó a lograr un lenguaje común y la apropiación de los conceptos principales promovidos por el programa y orientó la mejora de los servicios (consultorías, laboratorios, entrenamientos, investigación por contrato, etc.) para atender las necesidades del sector privado. Además, ayudo a sensibilizar sobre la necesidad de que la Universidad cumpla con esa misión importante de vincularse al sector productivo a través de la amplia base de expertos con que se cuenta. Así mismo, también se sensibilizó sobre la necesidad de asegurar calidad en los servicios que se están prestando a los sectores que así lo demanden. De igual forma, aunque poco a poco y no de forma homogénea en todas las universidades, se promovieron cambios en las estructuras institucionales con el fin de proporcionar un mejor entorno para la innovación, el espíritu empresarial y la colaboración en investigación, y contribuyó a proporcionar un marco común para organizar de mejor manera los intereses de investigación e innovación entre las universidades y el sector privado.

Los resultados obtenidos en las Universidades con el apoyo del PUE fueron múltiples, entre ellos destacan:

- Autoridades y académicos capacitados y sensibilizados sobre la necesidad de contar con una función de transferencia tecnológica.

- Vinculación de la investigación y la transferencia tecnológica de tal forma que los conocimientos lleguen a los beneficiarios de las Universidades.

- Promocion de la propiedad intelectual y el espíritu emprendedor en profesores y estudiantes, así como en el currículo, para convertir a las Universidades en más innovadoras.

- Evaluación de prácticas actuales en materia de investigación y extensión en las universidades.

- Desarrollo de mecanismos(sistemas de información) para hacer saber a la sociedad sobre lo que estamos haciendo en materia de investigación e innovación.

- Graduación de cinco nicaragüenses con grado de maestría y 25 académicos de las Universidades en gestión del capital intelectual

- Una ley de ciencia y tecnología e innovación presentada ante la asamblea nacional.

- Definición de políticas y normas sobre propiedad intelectual, innovación e investigación.

- Introducción de la gestión de la propiedad intelectual en la práctica universitaria.

- Definición de agendas de investigación en combinación con la industria local y la sociedad.

- Formación de profesores y líderes en innovación, propiedad intelectual y espíritu empresarial.

- Evaluación de los resultados de investigación de las universidades para establecer posibles oportunidades de negocios.

- Publicación de dos libros, uno que presenta los proyectos claves de innovaciones generadas por las Universidades CNU y un segundo que muestra un resumen de los logros importantes de las diez universidades que participaron en el Programa.

- Algunas Universidades iniciaron el proceso de protección del conocimiento generado. Por ejemplo, La UNA ha desarrollado un proceso orientado a identificar aquellos productos y servicios sujetos de protección.

- Productos y activos intangibles protegidos ante la oficina de registro de propiedad intelectual del MIFIC. 


\section{CONCLUSIONES Y RECOMENDACIONES}

El programa Universidad emprendedora tuvo impacto en la gestión de la colaboración en investigación entre actores claves del sistema de investigación e innovación. También permitió que las ideas difundidas a través del programa estén impregnando y contribuyendo a transformar las estructuras institucionales, logrando mejores formas de interactuar con otros actores claves y cambiando la actitud de los investigadores, autoridades universitarias, instituciones públicas y el sector privado, acerca de conceptos como la innovación, espíritu empresarial y colaboración para la investigación, entre otros. El programa también contribuyo a la necesidad de cambiar la imagen de la universidad, que hasta la fecha se le consideraba una entidad cerrada por la industria nicaragüense.

A la fecha, las universidades están mejor preparados para convertirse en universidades emprendedoras, como era el objetivo. El programa permitió mejora del entorno institucional para promover vínculos fuertes, y apoyar las innovaciones. Al respecto, en el libro "Innovaciones en Universidades Nicaragüenses" se mencionan una serie de iniciativas, las cuales aún están fragmentadas (Aleman, 2010)

El potencial y la iniciativa de las facultades, profesores y científicos para mejorar estos resultados son notables, pero existen otras limitaciones debido a problemas estructurales, tales como falta de recursos financieros para la investigación y la innovación, la debilidad y baja productividad del sector de las PYME, y el compromiso limitado de la gran empresa.

Antes esta problemática, las Universidades Nicaragüenses tienen que estar acorde a los retos que impone la sociedad del conocimiento, el PUE se constituyó en un punto de partida, marco el inicio de trasformaciones serias que permitirán a las Universidades llegar a ser emprendedoras. Aún hay un largo camino que recorrer, que incluye continuar integrando las políticas y procesos de innovación, integrar capacitación de innovación y emprendimiento en el currículo y continuar desarrollando competencias y procesos para la transferencia tecnología.
Hay que fortalecer las relaciones entre la Universidad, la sociedad e industria, mediante la formulación y ejecución de proyectos de colaboración conjuntos para solventar las necesidades locales, acceder a fondos, investigación contratada, proyectos de estudiantes, servicios, portafolio de resultados de investigación, derechos de propiedad intelectual, licenciamientos, etc.

En el caso de la Industria, se necesita evaluar las experiencias, necesidades y estratégicas para el futuro, fortalecer la calidad de la industria y el potencial para innovar, vincular la industria a universidades según necesidades y competencias, crear y evaluar proyectos pilotos, innovaciones de productos, organización y gestión, procesos, etc.

Las Universidades tienen que estar preparadas para desarrollar una formación (grado y postgrado), la investigación y los servicios basados en las necesidades de la sociedad e industria, el gobierno dispuesto para crear leyes y políticas que apoyen la innovación y facilitar las inversiones necesarias y la industria priorizar la innovación y co-financiar los servicios universitarios.

Finalmente, los impactos en términos de aumento de valor y los cambios económicos sólo pueden ser juzgados con el devenir del tiempo. Por lo tanto, es importante que las Universidades y sus socios puedan ejecutar revisiones anuales para ver cómo las capacidades generadas se desarrollan para producir los efectos deseados.

\section{REFERENCIAS BIBLIOGRÁFICAS}

Alänge, S; Scheinberg, S. 2005. InnovationSystemsin LatinAmerica, Sida, 95 paginas en la versión impresa.

Alemán, F; Medrano, H; Norgren, A; Reyes, A; Scheinberg, S. Editoress 2010. Innovaciones en las universidades nicaraguenses, CNU-SIDA-CIP. 322. p.

Johansson, M; Martin, L; Melander, F. 2009. How to Enhance Value of Research Results. UniversityIndustryCollaboration in Nicaragua, Master of ScienceThesis in Management and Economics of Innovations. ChalmersUniversity of Technology. Goteborg, Sweden, 2009; p. 5. 